\title{
Effect of Surface Oxygen Vacancy on Pt Cluster Adsorption and Growth on the Defective Anatase $\mathrm{TiO}_{2}(101)$ Surface
}

You Han ${ }^{1,2}$, Chang-jun Liu ${ }^{* 1}$, and Qingfeng Ge*²

${ }^{1}$ Key Laboratory of Green Chemical Technology, School of Chemical Engineering, Tianjin University, Tianjin 30072, China, and ${ }^{2}$ Department of Chemistry and Biochemistry, Southern Illinois University, Carbondale, Illinois 62901

\section{Supporting Information}

TABLE S1: Atomic Mulliken Charges and Charge Differences of Surface Atoms in Clean Defective Anatase $\mathrm{TiO}_{2}(101)$ Surface and in $\mathrm{Pt}_{1, \mathrm{Vac}}{ }^{*}$

\begin{tabular}{ccccc}
\hline & \multicolumn{2}{c}{ Defective } & & \\
\cline { 2 - 4 } & Frozen & Relaxed & & \\
& & & \\
& & & \\
& & & \\
& & & \\
5cTi(d) & 1.08 & 1.26 & 1.06 & 1.30 \\
& $(0.22)$ & $(0.04)$ & $(0.24)$ & \\
4cTi(d) & 1.13 & 1.34 & 1.13 & 1.36 \\
& $(0.23)$ & $(0.02)$ & $(0.23)$ & \\
3cO(d) & -0.65 & -0.70 & -0.66 & -0.64 \\
& $(0.01)$ & $(0.06)$ & $(0.02)$ &
\end{tabular}

${ }^{*}$ Charge differences in parentheses were calculated with respect to the charge of corresponding atoms on the perfect surface listed in the last column.

* Corresponding authors: changliu@public.tpt.tj.cn(C.-j. L.); qge@chem.siu.edu (Q.G.) 


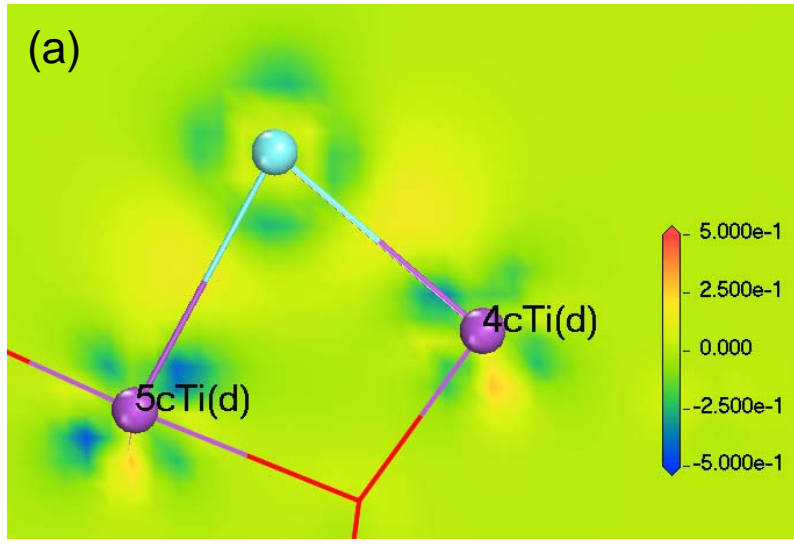

(b)

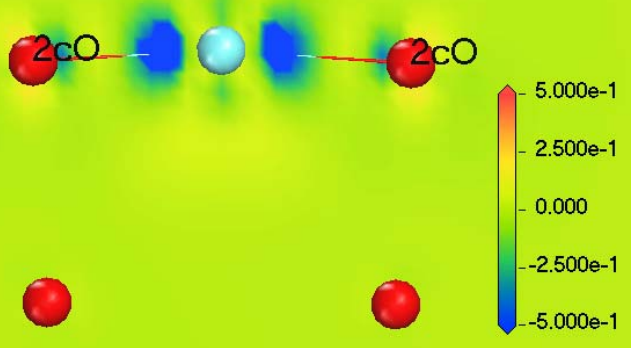

Figure S1. Electron density difference (EDD) contour maps for a Pt adatom in (a) $\mathrm{Pt}_{1, \mathrm{Vac}}$ and (b) $\mathrm{Pt}_{1, \mathrm{Bri}}$. In each case the reference density is the density sum of the Pt atom and the clean anatase $\mathrm{TiO}_{2}(101)$ in the respective configuration. 